\title{
Study on the Voltage Characteristic of Lithium Ion Battery during Rest Period after Charge
}

\author{
Lifu Li ${ }^{\mathrm{a}}$ and Dongyu Zhang ${ }^{\mathrm{b}, \text { * }}$ \\ Guangdong Key Laboratory of Automotive Engineering, South China University of Technology, \\ Guangzhou 510641, P.R China

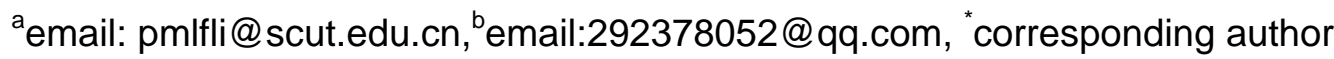

Keywords: Lithium Ion Battery; Rest; Voltage Model

\begin{abstract}
The open circuit voltage (OCV) of lithium ion battery is a key parameter for hybrid electric vehicle (HEV) to estimate the state-of-charge (SoC). And its instability will lead to big error of SoC estimation and have difficulty in implementing the dynamic control strategy of HEV, especially when the lithium ion battery rests after regenerative braking. According to the theory of electrochemistry, the lithium iron phosphate battery is studied in order to analyze the influence mechanism of lithium ion diffusion on the OCV when it rests after charge. And an OCV model appropriate for the rest period after charge is built by combining experiment with parameter identification method. The result of experiment shows that the OCV model is consistent with the OCV characteristic.
\end{abstract}

\section{Introduction}

The voltage characteristic of lithium ion battery is the precondition to match the vehicle power system and the key factor for both battery management system (BMS) design and SoC estimation[1]. According to the HEV theory, the batteries are in the alternating state of discharge, charge and rest when HEV experiences the process of acceleration, deceleration, and parking[2]. According to the theory of electrochemistry, the battery's voltage becomes unstable under the influence of electrode polarization, and it varies with time when the battery rests after charge, which will lead to big error of SoC estimation and have difficulty in implementing the dynamic control strategy of HEV. In order to solve these problems, an OCV model is established to improve the accuracy of SoC estimation and to ensure the implementation of the dynamic control strategy of HEV[3].

\section{The Voltage Characteristic Of Lithium Ion Battery}

According to the theory of electrochemistry, the positive reaction of the lithium ion battery (hereinafter referred to as LIB) in charge and discharge can be expressed as

$$
\mathrm{LiFePO}_{4} \underset{\text { discharge }}{\stackrel{\text { charge }}{\rightleftarrows}} \mathrm{Li}_{1-x} \mathrm{FePO}_{4}+x \mathrm{Li}^{+}+x e^{-}
$$

The negative one can also be expressed as

$$
x \mathrm{Li}^{+}+x e^{-}+6 \mathrm{C} \underset{\text { discharge }}{\stackrel{\text { charge }}{\rightleftarrows}} \mathrm{Li}_{x} \mathrm{C}_{6}
$$

Obviously, it can be seen from Eq.1 and Eq.2 that the LIB can make the transformation between electrical energy and chemical energy through the extraction and insertion of the lithium ion in its positive and negative electrode in the charge and discharge period. Fig. 1 shows the working principle of LIB. When the LIB is being charged, the lithium ions are extracted from the positive electrode, passing through the electrolyte and the separator, and finally inserted into the negative electrode. Meanwhile, the electrons are moved to the positive electrode through the external circuit[4]. Then the voltage between positive electrode and negative electrode varies with time. 


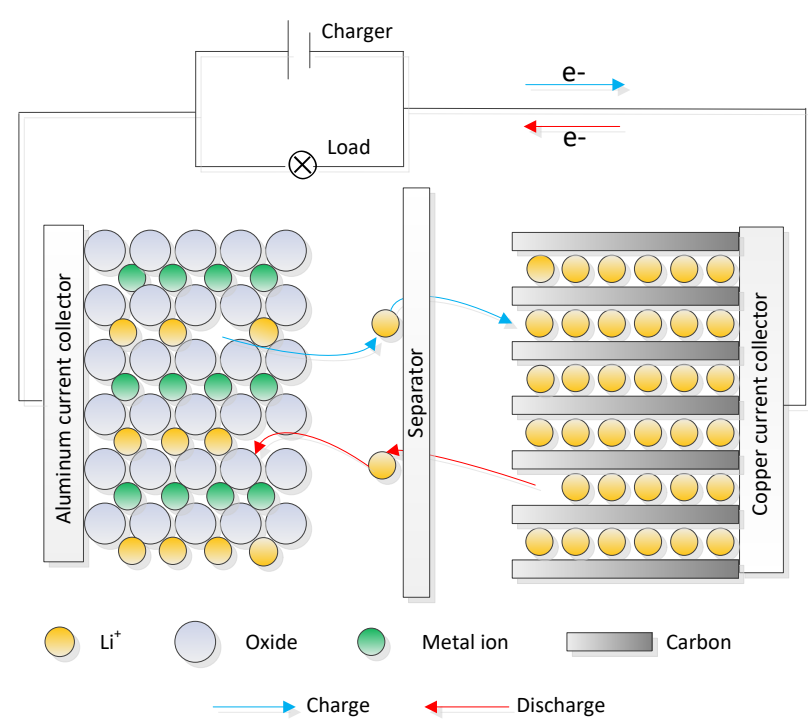

Fig. 1 the working principle of LIB

When the LIB rests after charge, the terminal voltage is regarded as OCV. It can be expressed as $U_{\mathrm{OCV}}(t)=\varphi_{+}(t)-\varphi_{-}(t)$

Where $\varphi_{+}(t)$ denotes the potential of LIB positive electrode, $\varphi_{-}(t)$ denotes the potential of LIB negative electrode. According to the theory of electrochemistry, $\varphi_{+}(t)$ and $\varphi_{-}(t)$ are related to the activity of the positive and negative materials. According to Nernst equation, the relation between $\varphi_{+}(t)$ and the activity of the substance can be expressed as

$$
\varphi_{+}(t)=\varphi_{+}^{\Theta}-\frac{\mathrm{RT}}{\mathrm{nF}} \ln \left(\frac{a_{\mathrm{LiFePO}_{4}}(t)}{a_{\mathrm{Li}_{1-x} \mathrm{FePO}_{4}}(t) a_{\mathrm{Li}^{+}}^{x}(t)}\right)
$$

And $\varphi_{-}(t)$ can also be expressed as

$$
\varphi_{-}(t)=\varphi_{-}^{\Theta}-\frac{\mathrm{R} T}{\mathrm{nF}} \ln \left(\frac{a_{\mathrm{Li}_{x} \mathrm{C}_{6}}(t)}{a_{\mathrm{Li}^{+}}(t) a_{\mathrm{C}}^{x}(t)}\right)
$$

Where $\varphi_{+}^{\Theta}$ represents standard oxidation-reduction potential of LiFePO4, $\varphi_{-}^{\Theta}$ represents standard oxidation-reduction potential of carbon, $\mathrm{T}$ denotes thermodynamic temperature, $\mathrm{R}$ denotes molar gas constant, $\mathrm{n}$ represents the electron number, and $\mathrm{F}$ denotes faraday constant. $a_{\mathrm{LiFePO}_{4}}(t)$, $a_{\mathrm{Li}_{1-x} \mathrm{FePO}_{4}}(t)$ and $a_{\mathrm{Li}^{+}}(t)$ are the activity of the substance of the positive electrode. $a_{\mathrm{Li}_{x} \mathrm{C}_{6}}(t)$, $a_{\mathrm{Li}^{+}}(t)$ and $a_{\mathrm{C}}(t)$ are the activity of the substance of the negative electrode. As the activity is difficult to be quantified, we can replace it with the proportion of lithium ion, such as the proportion of lithium ion in the positive electrode $x(t)$ and the proportion of lithium ion in the negative electrode $y(t)$. And both of them are the function of time. The positive electrode, negative electrode and their surfaces are considered as a whole, as the lithium ions on the electrode surfaces can also influence the potential of the electrodes. And the Eq.6 about the relation among $U_{\text {ocv }}(t)$, $x(t)$ and $y(t)$ can be derived from Eq.3.

$$
U_{\mathrm{OCV}}(t) \approx \varphi_{+}{ }^{\Theta}-\varphi_{-}{ }^{\Theta}+\mathrm{b}(x(t)-y(t))-\frac{\mathrm{R} T}{\mathrm{nF}} \ln \left(\frac{x(t)}{1-x(t)}\right)+\frac{\mathrm{R} T}{\mathrm{nF}} \ln \left(\frac{y(t)}{1-y(t)}\right)
$$


Where the parameter $\varphi_{+}^{\Theta}$ and $\varphi_{-}^{\Theta}$ can be calculated from the potential table of standard oxidation-reduction. The equation about $U_{\mathrm{OCV}}(t), x(t)$ and $y(t)$ in the room temperature $\left(25^{\circ} \mathrm{C}\right)$ can be derived by substituting parameter values of $\varphi_{+}^{\Theta}, \varphi_{-}^{\Theta}, \mathrm{R}, \mathrm{T}$ and $\mathrm{F}$ into Eq.6. When being charged, the lithium ion will be extracted out of the positive electrode and inserted into the negative electrode, which reduces $x(t)$ and increases $y(t)$. Then $U_{\text {ocv }}(t)$ will rise gradually during this period, because it is affected by $x(t)$ and $y(t)$.

According to the theory of electrochemistry, the variation of $x(t)$ and $y(t)$ are driven by the diffusion of the lithium ion on the surfaces of two electrodes when the LIB rests[5]. Fig. 2 shows the distribution of the ion. Fig. 2(a) reveals the distribution of the ion while the LIB is being charged. Fig. 2(b) displays the distribution of the ion when the LIB rests after charge .While the LIB is being charged, it leads to the decreases of the concentration of the reactant near the surface of the electrode or the increase of the concentration of the product, because the reactant is added slowly to the surface of the electrode or the product is spreading slowly from the surface of the electrode. So there is a concentration difference between the reactant concentration near the surface of the electrode and the concentration of the solution, which makes the potential of electrode deflected from its equilibrium potential[6]. When the LIB is rest after charge, the lithium ion on the surface of the electrode will make diffusion movement owing to the concentration difference, which leads to the decrease of $y(t)$ and the increase of $x(t)$.

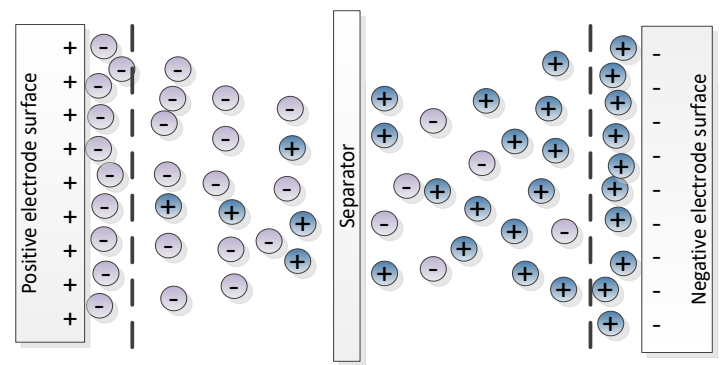

(a)Ion distribution when charging

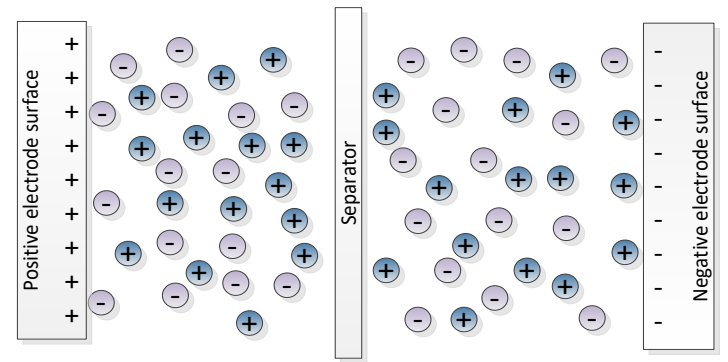

(b)Ion distribution when rest

$\oplus$ Positive ion $\odot$ Negative ion

Fig. 2 the distribution of the ion

Inferred from the Eq.6, there is phenomenon that OCV will drop when the LIB rests after charge. Fig. 3 shows the curve about electricity and voltage when LIB (10Ah) is being charged and rest. During the rest period, OCV shows a downtrend like section A-B. 


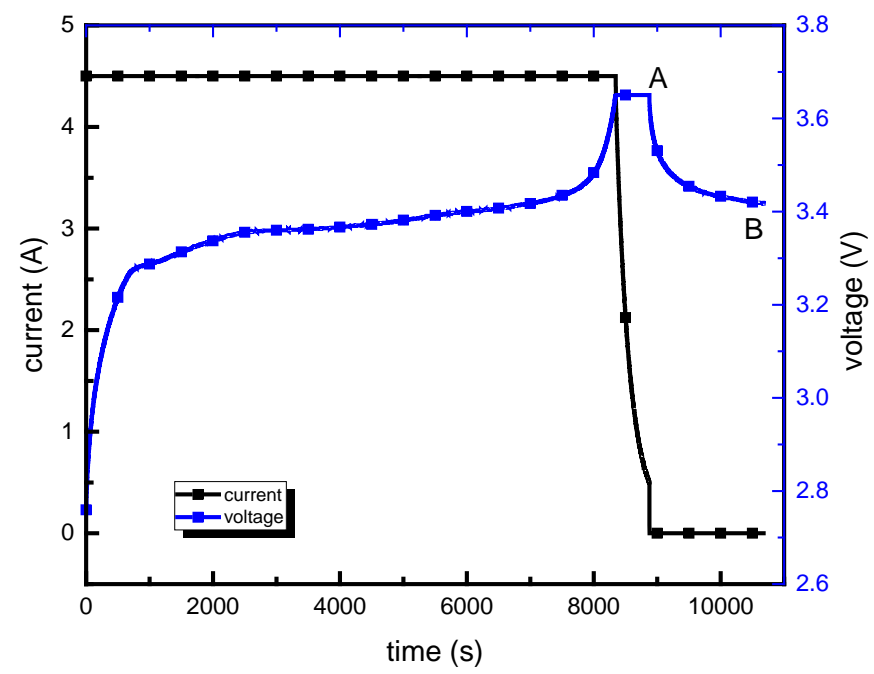

Fig. 3 the curve about electricity and voltage when LIB (10Ah) is charge and rest

According to the Fick's law, $J$ symbolizes the diffusion flux, defined as the mass of the lithium ions that pass the unit area perpendicular to the diffusion direction in unit time [7]. And it can be expressed as

$J=-\mathrm{D} \frac{\Delta C}{\Delta l}$

Where $D$ denotes diffusion coefficient, $C$ denotes concentration of the lithium ion, $l$ denotes the distance between the surface of electrode and the position with concentration $C$, $\Delta C / \Delta l$ denotes gradient in the direction of diffusion. The change rate of the lithium ion by diffusion cross section in the unit time is set as $\Delta n / \Delta t$.

$$
\frac{\Delta n}{\Delta t}=-\mathrm{D} \frac{\Delta C}{\Delta l} \frac{\mathrm{A}}{\mathrm{M}}
$$

Where A denotes the area of diffusion cross section, $\mathrm{M}$ denotes the molar mass of lithium. $\Delta n / \Delta t$ can be regarded as constant in Eq.8. Then $\Delta n_{x} / \Delta t$ and $\Delta n_{y} / \Delta t$ can be expressed as

$$
\left[\begin{array}{l}
\frac{\Delta x}{\Delta t} \\
\frac{\Delta y}{\Delta t}
\end{array}\right]=\left[\begin{array}{cc}
k_{x} & 0 \\
0 & k_{y}
\end{array}\right]\left[\begin{array}{c}
\frac{\Delta n_{x}}{\Delta t} \\
\frac{\Delta n_{y}}{\Delta t}
\end{array}\right]
$$

Where $\Delta n_{x} / \Delta t$ denotes the change rate of concentration of the lithium ion in the positive electrode, $\Delta n_{y} / \Delta t$ denotes the change rate of concentration of the lithium ion in the negative electrode, $k_{x}$ and $k_{y}$ are the proportionality coefficients. Then $x(t)$ and $y(t)$ can be expressed as

$$
\left[\begin{array}{l}
x(t) \\
y(t)
\end{array}\right]=\left[\begin{array}{l}
x_{0} \\
y_{0}
\end{array}\right]+\left[\begin{array}{l}
\frac{\Delta x}{\Delta t} \\
-\frac{\Delta y}{\Delta t}
\end{array}\right] t
$$

Where $x_{0}$ denotes the initial value of the proportion of lithium ion in the positive electrode after charge, $y_{0}$ denotes the initial value of the proportion of lithium ion in the negative electrode after charge. Based on Eq.6 and Eq.10, a OCV model can be expressed as 


$$
U_{\mathrm{OCV}}(t)=U_{0}-\Delta \varphi_{1} \ln \left(\frac{x_{0}-\frac{\Delta x}{\Delta t} t}{1-x_{0}+\frac{\Delta x}{\Delta t} t}\right)+\Delta \varphi_{2} \ln \left(\frac{y_{0}-\frac{\Delta y}{\Delta t} t}{1-y_{0}+\frac{\Delta y}{\Delta t} t}\right)
$$

Where $U_{0}$ denotes the terminal voltage without concentration polarization after charge, $\Delta \varphi_{1}$ denotes value of potential of positive electrode affected by lithium ion diffusion, $\Delta \varphi_{2}$ denotes value of potential of negative electrode affected by lithium ion diffusion.

In order to find the parameters of the OCV model under different charge conditions, the method of parameter identification of battery OCV model is proposed based on least square method according to the system identification principle[8]. The value of $U_{\mathrm{OCV}}(t)$ is measured in different charge conditions to get model parameter group $\theta$ on the basis ofEq.12.

$$
\min F(\theta)=\left\{\sum_{i=1}^{j}\left[\hat{U}_{O C V}\left(t_{i}\right)-U_{O C V}\left(t_{i}\right)\right]^{2}\right\}
$$

Where $\hat{U}_{O C V}\left(t_{i}\right)$ denotes the model estimations of the OCV, $U_{O C V}\left(t_{i}\right)$ denotes the experimental value, $j$ denotes the number of experimental values. Model parameter group $\theta$ includes $U_{0}, \Delta \varphi_{1}$, $\Delta \varphi_{2}, \quad x_{0}, \quad y_{0}, \quad \Delta x / \Delta t$ and $\Delta y / \Delta t$. The terminal voltage can be obtained accurately by the OCV model, which can improve the accuracy of SoC estimation.

\section{Experiment and Analysis}

In order to verify the characteristic of the voltage when the LIB rests after charge (SoC=1), a lithium iron phosphate battery (10Ah) is regarded as the experimental subject to identify the OCV model. The XINWEI Charge and Discharge Equipment (Equipment type : BTS-5V100A) is adopted as the experimental equipment to conduct the cycle charge and discharge test. Table 1 shows the experiment steps.

\begin{tabular}{|c|c|}
\hline number & Steps \\
\hline 1 & Rest for1(min) \\
\hline 2 & $\begin{array}{l}\text { Constant current discharge mode: } \\
\text { electricity is } 4500(\mathrm{~mA}), \\
\text { discharge cut-off voltage is } 2.5(\mathrm{~V})\end{array}$ \\
\hline 3 & Rest for 30(min) \\
\hline 4 & $\begin{array}{l}\text { Constant current charge mode: } \\
\text { electricity is } 4500 \text { (mA), } \\
\text { charge cut-off voltage is } 3.65(\mathrm{~V})\end{array}$ \\
\hline 5 & $\begin{array}{l}\text { Constant voltage charge mode: voltage } \\
\text { is } 3.65(\mathrm{~V}) \text {, charge cut-off electricity is } \\
500(\mathrm{~mA})\end{array}$ \\
\hline 6 & Rest for 30(min) \\
\hline 7 & $\begin{array}{l}\text { Constant current discharge mode: } \\
\text { electricity is } 4500(\mathrm{~mA}), \\
\text { discharge cut-off voltage is } 2.5(\mathrm{~V})\end{array}$ \\
\hline 8 & Rest for 30(min) \\
\hline
\end{tabular}

Table1 experiment steps

The terminal voltage, especially OCV value in the step 6, is recorded by experimental equipment according to the experiment steps. According to the system identification principle, the value of parameter $U_{0}, \Delta \varphi_{1}, \Delta \varphi_{2}, \quad x_{0}, y_{0}, \Delta x / \Delta t$ and $\Delta y / \Delta t$ can be obtained and are showed in Table 
2. Table2 Parameter value

\begin{tabular}{cccc}
\hline Parameter & Value & Parameter & Value \\
\hline$U_{0}$ & $3.38689(\mathrm{~V})$ & $y_{0}$ & 0.99821 \\
$\Delta \varphi_{1}$ & $0.02556(\mathrm{~V})$ & $\Delta x / \Delta t$ & $6.62855 \times 10^{-5}(1 / \mathrm{s})$ \\
$\Delta \varphi_{2}$ & $0.01004(\mathrm{~V})$ & $\Delta y / \Delta t$ & $9.52726 \times 10^{-5}(1 / \mathrm{s})$ \\
$x_{0}$ & $2.84815 \times 10^{-4}$ & & \\
\hline
\end{tabular}

The OCV model can be expressed as Eq.13 by substituting the parameter into the Eq.11. It shows the characteristics of lithium iron phosphate battery's voltage that varies with time.

$$
\begin{aligned}
\hat{U}_{O C V}(t)= & 3.38689-0.02556 \ln \left(\frac{0.0002848+0.0000663 \times t}{0.9997152-0.0000663 \times t}\right) \\
& +0.01004 \ln \left(\frac{0.99821-0.00009527 \times t}{0.99179+0.00009527 \times t}\right)
\end{aligned}
$$

Fig. 4 displays the curve of voltage obtained from experiment and OCV model. When the lithium iron phosphate battery rests after charge in the experiment, the decrease of voltage reaches $0.1 \mathrm{~V}$ in the first 200 seconds, and $0.08 \mathrm{~V}$ in the next 1600 seconds. The voltage dropping starts with a high rate but ends with a low rate regularly. The experimental values of the voltage are evenly distributed on both sides of the OCV model curve, which fits the characteristic of lithium iron phosphate battery's voltage[9].

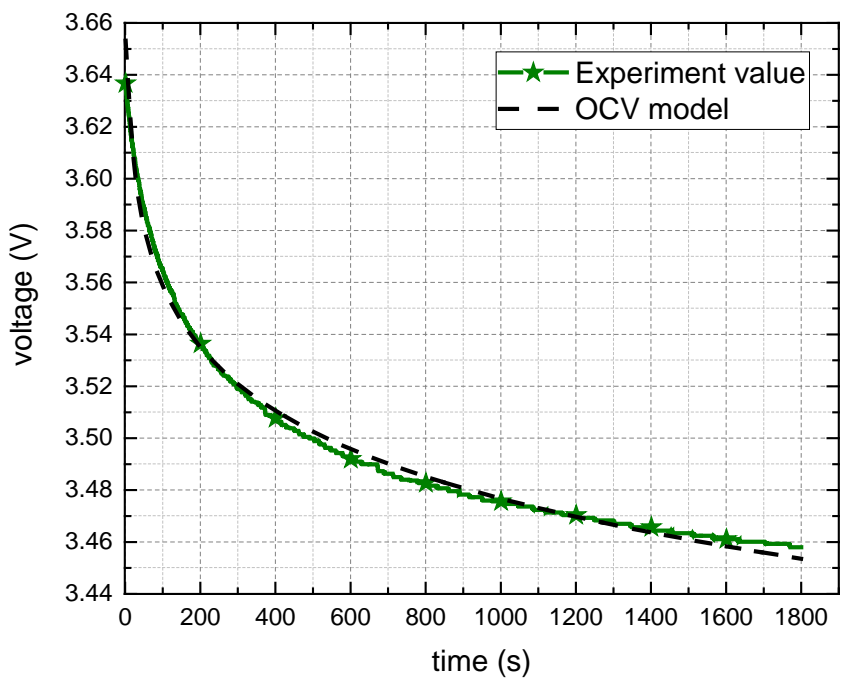

Fig. 4 the curve of voltage obtained from experiment and OCV model

\section{Conclusion}

The influence mechanism of lithium ion diffusion on the OCV when the battery rests after charge is analyzed to reveal the relation among OCV and the proportion of lithium ion in the positive electrode $x(t)$ and the proportion of lithium ion in the negative electrode $y(t)$. An OCV model appropriate for the rest period after charge is built by combining experiment with parameter identification method. The result of experiment shows that the OCV model is consistent with the OCV characteristic. And the model is of vital importance to improve the accuracy of SoC estimation. 


\section{Acknowledgement}

This study is sponsored by the fund for construction of public welfare research and ability of Guangdong Province. The project title is "The prediction and control technology for cycle life of lithium iron phosphate power battery based on driving conditions” (NO.2014B010106004).

\section{Reference}

[1] Hannan M A, Lipu M S H, Hussain A, et al. A review of lithium-ion battery state of charge estimation and management system in electric vehicle applications: Challenges and recommendations[J]. Renewable \& Sustainable Energy Reviews, 2017, 78:834-854.

[2] Kim H, Kang G S. Scheduling of Battery Charge, Discharge, and Rest[C]// IEEE Real-Time Systems Symposium. IEEE Computer Society, 2009:13-22.

[3] Hu C, Youn B D, Chung J. A multiscale framework with extended Kalman filter for lithium-ion battery SOC and capacity estimation[J]. Applied Energy, 2012, 92(4):694-704.

[4] Christian Julien, Alain Mauger, Ashok Vijh, et al. Lithium Batteries[M]. Switzerland: Springer International Publishing, 2016: 75-76.

[5] Park M, Zhang X, Chung M, et al. A review of conduction phenomena in Li-ion batteries[J]. Journal of Power Sources, 2010, 195(24): 7904-7929.

[6] Xi X, Li X, Wang C, et al. Impact of Proton Concentration on Equilibrium Potential and Polarization of Vanadium Flow Batteries[J]. Chempluschem, 2015, 80(2):382-389.

[7] Dubey A M, Ram R, Yadav A K. Ion cell performance using single particle representation of battery electrode[J]. Journal of Electroanalytical Chemistry, 2016, 768:11-17.

[8] Rahimi-Eichi H, Baronti F, Chow M Y. Online Adaptive Parameter Identification and State-of-Charge Coestimation for Lithium-Polymer Battery Cells[J]. IEEE Transactions on Industrial Electronics, 2014, 61(4):2053-2061.

[9] Dharmakeerthi C H, Mithulananthan N, Saha T K. Impact of electric vehicle fast charge on power system voltage stability[J]. International Journal of Electrical Power \& Energy Systems, 2014, 57(5):241-249. 